\title{
TRAUMATIC INJURY TO THE HEART DUE TO BLUNT FORCE \\ With a case report
}

By B. S. DHILLON, F.R.C.S. (Edinburgh), F.R.C.S. (England)

Royal Infirmary, Sheffield

Non-penetrating injuries of the chest wall can give rise to a variety of cardiac lesions. The extent of cardiac damage depends on the intensity of the injury and on pre-existent disease of the heart. In the majority of cases there is a direct blow to the anterior chest wall but serious myocardial damage has been produced by blows directed against the posterior chest wall and abdomen. The crushing chest accidents account for a large number of reported cases but a blow in the precordium with fist, cricket ball, hockey ball, baseball and kick by a powerful animal can produce heart injury. There may or may not be an associated fracture of the ribs; greater cardiac damage seems to occur in patients without associated fracture of the ribs. ${ }^{1}$ It is difficult to assess the exact incidence of cardiac injury as most patients with minor damage pass unnoticed or there is reluctance to make the diagnosis. The post-mortem incidence of cardiac involvement in instances of blunt trauma to the chest is 18 to 26 per cent. ${ }^{2}$ A frequent cause of cardiac injury is "steering wheel" accidents.

The pericardium and myocardium are most frequently injured but valves and their attachments have also been torn following blunt trauma to the chest wall. Contusion of the pericardium passes unnoticed as it rarely produces any signs and symptoms. Myocardial damage may amount to superficial contusion of any chamber of the heart, it may be partial laceration with or without injury to the coronary vessels or it may produce complete rupture of one or more chambers of the heart with instananeous death. Bright and Beck ${ }^{3}$ have collected $\mathrm{I} 3$ cases with perforation of the interventricular septum and one case of rupture of the inter atrial septum. It is quite possible that, in rare instances, the initial tear may involve the endocardium ${ }^{4}$ only with subsequent development of an aneurysm and delayed rupture of the heart. There are well authenticated cases in the medical literature of rupture of normal heart valves as a result of blunt injury to the chest wall. The aortic valve is most frequently affected and injury to the mitral valve includes tear of the chordae tendinae more commonly, tear of the valve cusps itself and occasionally tear of a papillary muscle. ${ }^{6}$
The anterior aspect of the heart is injured most frequently, which is in direct relation to the site of the mechanical force, but the posterior aspect of the heart may be bruised by squeezing against the vertebral column. The sudden increase of the intracardiac pressure produced by mass displacement of the blood towards the heart can cause tearing of the valves and their attachments, rupture of the auricles and in rare cases of the ventricles. ${ }^{7}$ The delayed rupture of the heart has been known to occur and the usual time is 7-I4 days after injury. It usually follows deep contusion (or infarction) of the wall with subsequent softening and rupture. ${ }^{8}$ The delayed rupture of the chordae tendinae has not been reported before, this is especially liable to happen if the papillary muscles or chordae tendinae have been traumatised at the time of the original injury and the onset of rapid auricular fibrillation a few days later produces a marked strain on the already weakened valve attachments, leading to their rupture.

The case history of a patient is presented who sustained injury to the heart following a crushing injury to the chest.

\section{Case History}

A male patient aged 30 was admitted to hospital at 4 p.m. on 25.8 .56 . He was working in a trench $12 \mathrm{ft}$. deep when a fall of clay and rock trapped him against the skip, crushing his left chest. On examination, he was a well-built man complaining of severe pain in the left chest. Pulse I10, fair volume, respiration 30 per minute and B.P. 100/55. $\mathrm{He}$ had a large haematoma over the anterior ends of the left 3 rd and $4^{\text {th }}$ ribs, another haematoma below the left scapula and surgical emphysema of the left chest wall posteriorly. The trachea was central, apex beat could be felt in the $5^{\text {th }}$ interspace in the anterior axillary line, heart sounds were clearly audible and no murmur was detected. The patient was extremely distressed with pain, morphia, $\frac{1}{4}$ gr., was given intravenously and circular strapping (elastoplast) applied from the level of the 2 nd ribs to the level of the 9 th ribs, with immediate relief of pain. At 4.30 p.m. a 


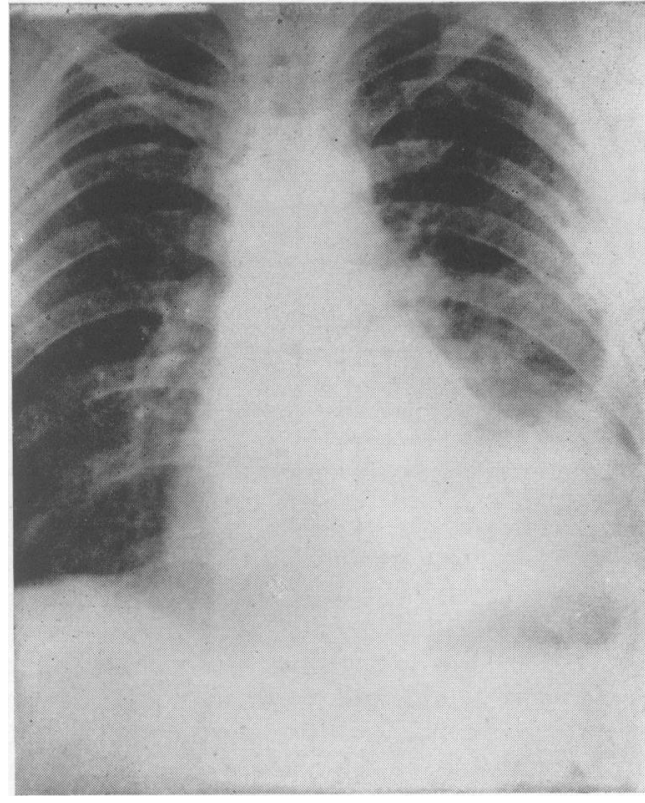

Fig. 1.-X-ray chest on admission showing small left haemothorax.

radiograph of the chest was taken and the patient transferred to the thoracic surgical ward. B.P. and pulse were recorded every $I_{5}$ minutes and by 5 p.m. B.P. was $80 / 55$ and pulse I 20 per minute.

Serial B.P. readings were recorded which showed continued fall in the systolic blood pressure and by 8 p.m. it was $60 \mathrm{~mm}$. of $\mathrm{Hg}$. while diastolic blood pressure stayed at a more or less constant level $(55 \mathrm{~mm}$. of $\mathrm{Hg})$. There was a steady rise in the pulse rate and by 8 p.m. it was noticed that there was a rise in the jugular venous pressure. At 8.30 p.m. intravenous blood transfusion was commenced and two more radiographs of the chest were taken, which showed progressive increase in the cardiac size and considerable effusion in the left pleural cavity. The heart sounds were muffled and by 9 p.m. there was marked distension of the jugular veins. E.C.G. showed ventricular extrasystoles and inversion of $T_{1}$. From the ahove data it was concluded that the patient had hatemopericardium which had resulted in cardiac tamponade and progressive bleeding in the left pleural cavity.

9 p.m. Pericardial tap via the xiphi-sternal route was performed and i 50 c.c. of blood aspirated from the pericardium, following which there was marked diminution in the size of the distended jugular veins, and some improvement in the general condition of the patient. At 9.30 p.m. the B.P. was $60 / 55$ and it was decided to carry out thoracotomy to control the bleeding points.

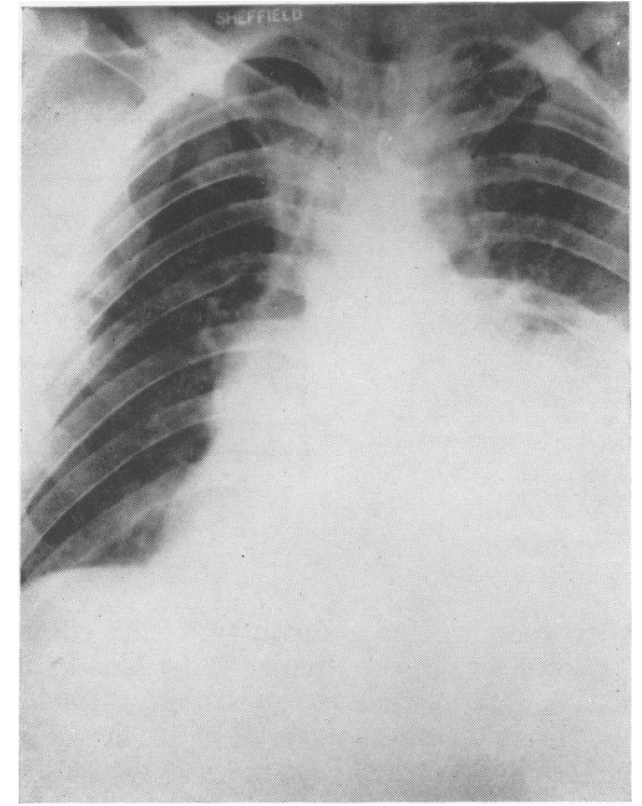

FIG. I I.-X-ray chest two hours after admission showing increase in the left haemothorax and increase in the cardiac size.

I0.30 p.m. Left Thoracotomy (B.S.D.) under G.A. The patient was placed in the right lateral position and a postero-lateral incision was made. After the skin and muscles were incised it was noticed that the intercostal muscles of the left 6 th interspace had been completely torn by the injury and the lung was presenting through this extensive gap in the 6th interspace, there was fracture of the anterior ends of the 2 nd, 3 rd and $4^{\text {th }}$ ribs and a double fracture involving the $5^{\text {th }}$, 6 th and 7 th ribs. There were nearly 3 pints of blood inside the pleural cavity, which was rapidly sucked out and the bleeding appeared to be coming from three sources. (I) From the ruptured intercostal vessels of the 3 rd and 4 th spaces. The torn ends of these vessels were freely hanging in the pleural cavity. (2) From the torn intercostal muscles of the 6th interspace. (3) From a tear in the left lower lobe near the inferior pulmonary vein.

Bleeding from the intercostal vessels and the lung was rapidly controlled. There was a large haematoma along the phrenic nerve, which completely covered the upper half of the pericardium and the ascending, transverse and descending part of the arch of aorta was surrounded by suffused blood. The pericardium was tense and full of blood, and it was noticed that the wall of the left auricle was contused and this contusion extended along the atrio venticular groove on to the wall of 


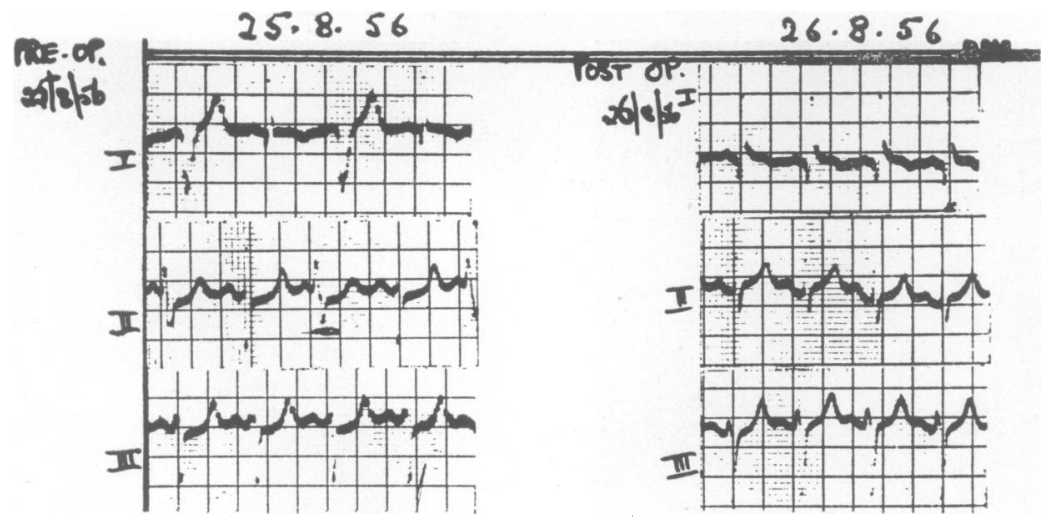

Fig. 3.-25.8.56. Preoperative E.C.G. showing ventricular extrasystoles and inverted $\mathrm{T}_{1}$. 26.8.56. Postoperative E.C.G. showing sinus rhythm.

the left ventricle for about 2 in. The coronary arteries and veins were intact. The blood in the pericardium appeared to be coming from this extensive contusion of the wall of the left auricle and left ventricle which was more of the nature of generalized oozing than a specific bleeding point. A hole in the pericardium was left for drainage into the pleural cavity. The fractured ends of the ribs were trimmed and an appreciable gap left between the rib ends to reduce the post-operative pain.

The pleural cavity was drained-an intercostal catheter connected to underwater seal. $\mathrm{He}$ was transfused with 1,800 c.c. of blood during the operation.

\section{Post-operative Progress}

On return to the ward the pulse was 80 , regular, good volume, B.P. I20/80 and no cardiac murmur was detected except for a pericardial rub, which is not unusual after the pericardium has been opened.

26.8.56-4 p.m. The patient was listless, tongue was dry, pulse I 30 , poor volume, extremities were cold and B.P. I05/70. There was no obvious loss of fluid to account for this circulatory collapse, examination of the abdomen revealed slight distension in the epigastric region, marked tympanitic note on percussion and a portable radiograph of the abdomen revealed acute dilatation of the stomach. A Ryles tube was passed into the stomach and $r, 45^{\circ}$ c.c. of dark-brown fluid aspirated from the stomach. An adequate electrolyte and fluid replacement was carried out with improvement in the general condition of the patient.

27.8.56. The routine post-operative examination of the cardiovascular system revealed no abnormality.

28.8.56. The patient maintained satisfactory improvement but at I p.m. there was sudden onset of auricular fibrillation with apex rate of 160 and B.P. I0o/60, the extremities were cold and the pulse was irregular and weak in volume (rate?). The auricular fibrillation was paroxysmal first but soon became fully established. The patient was digitalized with digoxin $1.5 \mathrm{mg}$. followed by digoxin I mg. at 6-hourly intervals for two doses and maintained on digoxin $0.25 \mathrm{mg}$. t.i.d.

29.8.56. There was rise in the jugular venous pressure with crepitations at the right base and sacral oedema. A systolic thrill could be palpated in the mitral areas and a harsh systolic murmur Grade III, conducted to the axillae was heard in the mitral area. The congestive cardiac failure responded to restricted fluid intake, salt-free diet and Mersalyl injections. His further postoperative progress was smooth.

On I.10.56 examination of the heart revealed apex beat in the 6th space in the anterior axillary line.

Mitral Area. A systolic thrill was still palpable, the first sound was normal, there was a loud, harsh systolic murmur (Grade III) conducted to the axilla. Fluoroscopic examination of the heart revealed enlargement of the left auricle and left ventricle. He was discharged home with instructions to attend the follow-up clinic.

\section{Discussion}

This case presents several interesting features. The development of haemopericardium following closed injury of the chest is definite evidence of myocardial damage or injury to the coronary vessels. It may be several hours before there is evidence of cardiac tamponade, if the bleeding is slow and moderate as in this case. The increased intrapericardial pressure interferes with normal filling of the auricles from the venous side, it reduces the cardiac output which produces a fall in blood pressure, narrowing of the pulse pressure 
and rise in the venous pressure. The pulse is small and its paradoxical nature can be more easily elicited with sphygmomanometer than by palpation. Cardiac tamponade can be the cause of continued shock and there may be no objective evidence of distended neck veins but manometric estimation of the venous pressure always shows a rise above the normal value. It is hardly possible to conduct fluoroscopic examination in a seriouslyill patient but repeated portable radiographs of the chest can yield useful information if the cardiac silhouette is progressively enlarging. Pericardial aspiration via the xiphisternal route is a comparatively easy procedure which helps to establish the diagnosis and in some cases repeated aspiration of the pericardium may be all that is necessary to relieve the acute cardiac tamponade. However, if the patient's condition remains unchanged or deteriorates after improvement, operative interference with repair of the wound should be carried out. It is clear from the operative findings in this case that repeated aspiration of the pericardium would have been sufficient to relieve the cardiac tamponade but progressive bleeding from the intercostal vessels and " bursting " of the left 6 th intercostal space with herniation of the lung, though the skin and chest wall muscles were intact, were the main reasons for carrying out left thoracotomy. It is easy to miss the traumatic rupture of the parietal pleura and the intercostal muscles, the patient usually comes back several weeks later with bulging and pain due to herniation of the lung. If recognized early it should be repaired as soon as the patient is fit enough to stand the operation.

The most notable feature about this case was the development of a systolic thrill and harsh systolic murmur in the mitral area following sudden onset of auricular fibrillation three days after injury. This is very suggestive of delayed rupture of the chordae tendinae of the mitral valve, resulting in pure mitral incompetence. There was no detectable cardiac murmur at the time of his admission and no murmur was detected on the first or second day following injury when the apex rate was between 80 to 90 per minute. The previous history of the patient was, singularly, negative.

It is unlikely that he sustained rupture of a papillary muscle of the mitral valve, as the subsequent progress would have been rapidly downhill.

The fluoroscopic examination of the heart at the time of the patient's discharge from the hospital (five weeks after injury) showed enlargement of the left auricle and left ventricle (supported by E.C.G.).

The onset of auricular fibrillation is not unusual following cardiac trauma due to non-penetrating injury of the chest. ${ }^{8}$ In this patient there was spontaneous reversion to normal rhythm about three weeks after the onset of auricular fibrillation.

Acute dilatation of the stomach and intestinal ileus may supervene with dramatic rapidity after chest injury. Cameron et al. ${ }^{9}$ have reported an incidence of 16.5 per cent. following severe injury to the chest. The acute dilatation of the stomach is liable to be missed in the initial stages if this possibility is not kept in mind. The patient is usually listless and there is general deterioration in the cardio-respiratory status, followed by anorexia and epigastric fullness; copious vomiting takes some time to develop. A gastric tube can produce dramatic relief.

\section{Summary}

I. The case history of a patient is reproduced who sustained contusion of the left auricle, the left ventricle and the pericardium following a nonpenetrating injury to the chest. These injuries to the heart produced extravasation of blood inside the pericardium, resulting in cardiac tamponade.

2. He also sustained complete disruption of the intercostal muscles of the left sixth interspace and progressive bleeding from the intercostal arteries:

3. On the first post-operative day he developed acute dilatation of the stomach which presented with symptoms of dehydration and circulatory coilapse.

4. Nearly 63 hours after operation there was sudden onset of auricular fibrillation with apex rate of 160 per minute. Following this a harsh systolic murmur Grade III was heard in the mitral area for the first time. It is assumed that onset of rapid auricular fibrillation produced rupture of the chordae tendinae, which must have been traumatized and weakened at the time of original injury.

\section{Acknowledgments}

I wish to thank Mr. A. W. Fawcett for permission to publish this paper.

\section{REFERENCES}

r. ARENBURG, H. (1943), Ann. intern. Med., 19, 326.

2. MESSEN, H. (1955), Arch. klin. Chir., 282, 288.

3. BRIGHT, E. F., and BECK, C. S. (1935), Amer. Heart $\mathcal{f}$., IO, 293 .

4. BARBER, H. (1940), Brit. med. F., 520.

5. KISSANE, R. W., FIDLER, R. S., and KOONS, R. A. (1937), Ann. intern. Med., II, 907 .

6. GLENDY, R. E., and WHITE, P. D. (1936), Amer. Heart. F., II, 366 .

7. GROSSE BROCKHOFF, F. (1955), Arch: klin. Chir., 282, 300.

8. WARBURG, E. (1940), Brit. Heart $\%$., 11, 271.

9. CAMERON, D. A., O'ROURKE, P. V., and BURT, C. W: (1949), Amer. 今. Surg., 78, 688. 